\title{
ON THE SCHULTZ POLYNOMIAL AND HOSOYA POLYNOMIAL OF CIRCUMCORONENE SERIES OF BENZENOID
}

\author{
MOHAMMAD REZA FARAHANI
}

\begin{abstract}
Let $G=(V, E)$ be a simple connected graph. The sets of vertices and edges of $G$ are denoted by $V=V(G)$ and $E=E(G)$, respectively. In such a simple molecular graph, vertices represent atoms and edges represent bonds. The distance between the vertices $u$ and $v$ in $V(G)$ of graph $G$ is the number of edges in a shortest path connecting them, we denote by $d(u, v)$.

In graph theory, we have many invariant polynomials for a graph $G$. In this paper, we focus on the Schultz polynomial, Modified Schultz polynomial, Hosoya polynomial and their topological indices of a molecular graph circumcoronene series of benzenoid $H_{k}$ and specially third member from this family. $H_{3}$ is a basic member from the circumcoronene series of benzenoid and its conclusions are base calculations for the Schultz polynomial and Hosoya polynomial of the circumcoronene series of benzenoid $H_{k}$ $(k \geq 3)$.
\end{abstract}

AMS Mathematics Subject Classification : 05C12, 05A15.

Key words and phrases: Molecular graph, Schultz polynomial, Modified Schultz polynomial, Schultz index, Modified Schultz index, Hosoya polynomial, Wiener index, Circumcoronene, Benzenoid.

\section{Introduction}

Let $G=(V, E)$ be a simple connected graph. The sets of vertices and edges of $G$ are denoted by $V=V(G)$ and $E=E(G)$, respectively. In such a simple molecular graph, vertices represent atoms and edges represent bonds.

The distance between the vertices $u$ and $v$ in $V(G)$, of graph $G$ is the number of edges in a shortest path connecting them, we denote by $d(u, v)$. An edge $u v$ of graph $G$ is joined between two vertices $u$ and $v(d(u, v)=1)$. Since $G$ is connected, $d(u, v)$ exists for all vertices $u, v \in V(G)$. In this paper, the number

Received July 31, 2012. Revised December 20 2012. Accepted January 23, 2013.

(c) 2013 Korean SIGCAM and KSCAM. 
of adjacent vertices with $v$ in $V(G)$ is degree of vertex $v$ and denote by $\delta_{v}$, that is an important terminology in graph theory.

In chemical, physics and nano sciences, we have many different family of molecular graph. One of the appealing family is the molecule benzene and the circumcoronene series of benzenoid, $H_{k}, k \geq 1$, (see Figure 3 and references [44]). The molecule benzene is more practical in the chemical, physics and nano sciences. In Figure 1, the first three graphs from the circumcoronene series of benzenoid, $H_{k}$, are shown Also is very useful to create the aromatic compounds. See reference $[7,8,13,16,44]$.
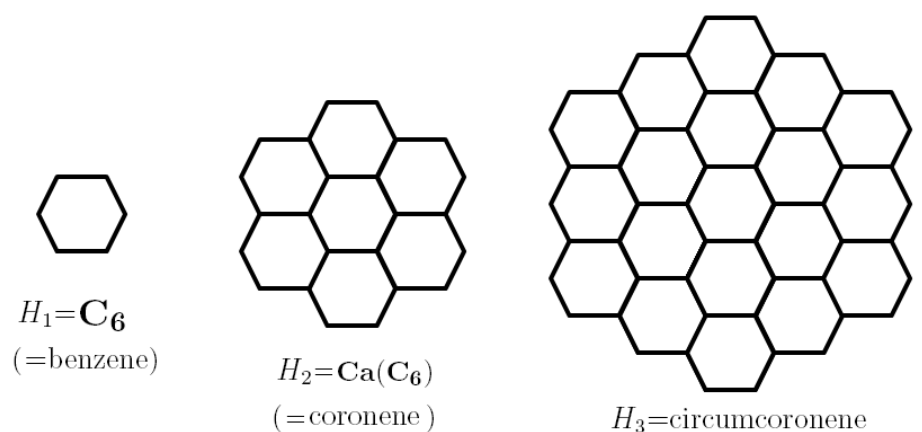

Figure 1. The first three graphs $H_{1}, H_{2}$ and $H_{3}$ from the circumcoronene series of benzenoid, such that $H_{1}, H_{2}$ are graphs $C_{6}$ and the Capra of planar benzenoid $\mathrm{Ca}\left(C_{6}\right)$, respectively.

In this paper, we focus on the third member of this family, namely graph $H_{3}$ in Figure 1, which coronene and benzene denoted by $H_{2}$ and $H_{1}=C_{6}$, respectively.

Also, in graph theory, we have many invariant polynomials for a graph, that they have usually integer coefficients. A polynomial of a graph is related with construction of graph, which is invariant under graph automorphisms. It is easy to see that every polynomial defines a topological index.

In this paper, we compute the Schultz polynomial, Modified Schultz polynomial and Hosoya polynomial of molecular graph circumcoronene $H_{3}$ and obtain their topological indices. The Schultz polynomial was introduced by H.P. Schultz in 1989 for the molecular topological index [46] and Modified Schultz index was defined by S. Klavzar and I. Gutman in 1997 [42]. The Hosoya polynomial was introduced by H. Hosoya, in 1988 [31].

The molecular topological index studied in many papers $[12,17,20,42,47]$ and computed $[1,6,9,10,14,15,21-28,32-39,42,46]$. In a series of papers, the Schultz indices of some nanotubes computed [20, 32-39, 41, 43, 45, 50]. The Hosoya polynomial and Wiener index of some molecular graph computed [3, $4,5,11,15,18,29-31,40,48,49,51,52]$. We denoted the Schultz polynomial by $S c(G, x)$, the Modified Schultz polynomial by $S c^{*}(G, x)$ and the Hosoya 
polynomial by $H(G, x)$. The Schultz polynomial of graph $G$ is defined as:

$$
S c(G, x)=\frac{1}{2} \sum_{u, v \in V(G)}\left(\delta_{u}+\delta_{v}\right) x^{d(u, v)}
$$

And its topological index is:

$$
S c(G)=\frac{1}{2} \sum_{u, v \in V(G)}\left(\delta_{u}+\delta_{v}\right) d(u, v)=\left.\frac{\partial(S c(G, x))}{\partial x}\right|_{x=1}
$$

The Modified Schultz polynomial of $G$ is defined as:

$$
S c^{*}(G, x)=\frac{1}{2} \sum_{u, v \in V(G)}\left(\delta_{u} \times \delta_{v}\right) x^{d(u, v)}
$$

And its topological index is:

$$
S c^{*}(G)=\frac{1}{2} \sum_{u, v \in V(G)}\left(\delta_{u} \times \delta_{v}\right) d(u, v)=\left.\frac{\partial\left(S c^{*}(G, x)\right)}{\partial x}\right|_{x=1}
$$

Definition $1.1([15])$. Let $d(u, v)=i$ is distance between vertices $u$ and $v$ of $G$. Then, $D_{i}=\{(u, v) \mid u, v \in V(G), d(u, v)=i\}$ is the set of some dual $(u, v)$ of vertex set $V(G)$.

In this paper, we denoted the size of $D_{i}$ by $d(G, i)\left(=\left|D_{i}\right|\right)$. The Hosoya polynomial of $G$ is defined as:

$$
H(G, x)=\sum_{u, v \in V(G)} x^{d(u, v)}=\sum_{i=0}^{d(G)} d(G, i) x^{i}
$$

with $d(G, 0)=n$ and $d(G, 1)=e$, such that $n$ is the number of vertices in the graph $G$, e the number of edges and $d(G)$ the topological diameter (i.e. the longest topological distance in $G$ ). See reference [11]. One of very important topological index in graph theory is Wiener index, that obtain from Hosoya polynomial and is equal to:

$$
W(G)=\frac{1}{2} \sum_{u \in V(G)} \sum_{v \in V(G)} d(u, v)=\left.\frac{\partial(H(G, x))}{\partial x}\right|_{x=1}=\sum_{i=0}^{d(G)} i . d(G, i)
$$

We were computed the Schultz polynomial and Modified Schultz polynomial of coronene $\mathrm{H}_{2}=\mathrm{Ca}\left(\mathrm{C}_{6}\right)$, (the second member of benzenoid family or circumcoronene series) in the following theorem.

Theorem $1.2([15])$. Let $H_{2}=C a\left(C_{6}\right)$ be the Capra of planar benzenoid (or coronene). Then:

- The Schultz polynomial of $\mathrm{H}_{2}$ is equal to:

$$
S c\left(H_{2}, x\right)=156 x+252 x^{2}+294 x^{3}+276 x^{4}+222 x^{5}+132 x^{6}+48 x^{7}
$$


So the Schultz index of $\mathrm{H}_{2}$ is $\mathrm{Sc}\left(\mathrm{H}_{2}\right)=4884$.

- The Modified Schultz polynomial of $\mathrm{H}_{2}$ is equal to:

$$
S c^{*}\left(H_{2}, x\right)=204 x+330 x^{2}+381 x^{3}+348 x^{4}+267 x^{5}+144 x^{6}+48 x^{7}
$$

So the Modified Schultz index of $\mathrm{H}_{2}$ is $\mathrm{Sc}^{*}\left(\mathrm{H}_{2}\right)=5934$.

- The Hosoya polynomial of $\mathrm{H}_{2}$ is equal to:

$$
H\left(H_{2}, x\right)=24+30 x+48 x^{2}+57 x^{3}+54 x^{4}+45 x^{5}+30 x^{6}+12 x^{7}
$$

Also, the Wiener index of $H_{2}$ is $W\left(H_{2}\right)=1002$ ([19, 44, 49]).

Now, in following theorems, we compute the Schultz polynomial, Modified Schultz polynomial and Hosoya polynomial of third member from the circumcoronene series of benzenoid.

Theorem 1.3. Let $H_{3}$ be the circumcoronene of benzenoid series. Then:

- The Schultz polynomial of $\mathrm{H}_{3}$ is equal to:

$$
\begin{aligned}
& S c\left(H_{3}, x\right)=396 x+696 x^{2}+912 x^{3}+1026 x^{4}+1062 x^{5} \\
& +1008 x^{6}+894 x^{7}+714 x^{8}+516 x^{9}+300 x^{10}+108 x^{11}
\end{aligned}
$$

So the Schultz index of $\mathrm{H}_{3}$ is $\mathrm{Sc}\left(\mathrm{H}_{3}\right)=40788$.

- The Modified Schultz polynomial of $\mathrm{H}_{3}$ is equal to:

$$
\begin{aligned}
& S c^{*}\left(H_{3}, x\right)=546 x+966 x^{2}+1263 x^{3}+1410 x^{4}+1449 x^{5} \\
& +1356 x^{6}+1182 x^{7}+918 x^{8}+336 x^{9}+336 x^{10}+108 x^{11}
\end{aligned}
$$

So the Modified Schultz index of $\mathrm{H}_{3}$ is $\mathrm{Sc}^{*}\left(\mathrm{H}_{3}\right)=53502$.

Theorem 1.4. Let $\mathrm{H}_{3}$ be the circumcoronene. Then, the Hosoya polynomial of $\mathrm{H}_{3}$ is equal to:

$$
\begin{aligned}
& H\left(H_{3}, x\right)=54+72 x+126 x^{2}+165 x^{3}+186 x^{4}+195 x^{5} \\
& \quad+186 x^{6}+168 x^{7}+138 x^{8}+102 x^{9}+66 x^{10}+27 x^{11}
\end{aligned}
$$

Also, the Wiener index of $H_{3}$ is $W\left(H_{3}\right)=7809$.

The molecular graph $H_{3}$ is a basic member from the circumcoronene series of benzenoid. Its conclusions is main calculations for the Schultz polynomial, Modified Schultz polynomial, Hosoya polynomial of the circumcoronene series of benzenoid $H_{3}$. So in continue, we present some result for the Schultz polynomial, Modified Schultz polynomial and Hosoya polynomial of general case of circumcoronene series of benzenoid, $H_{k}, \forall k \in \mathbb{N}$ and determine several sentence of favorite polynomial of this family. The Theorem 1.2, Theorem 1.3, Theorem 1.4, and Theorem 1.5 are the main results in this paper.

Theorem 1.5. Let $H_{k}(\forall k \geq 2)$ be the circumcoronene series of benzenoid, such that $\left|V\left(H_{k}\right)\right|=6 k^{2}$ and $\left|E\left(H_{k}\right)\right|=9 k^{2}-3 k$. Then the first, second and third sentence of Hosoya Polynomial, Schultz Polynomial and Modified Schultz Polynomial of $G$ are listed as follow:

I. The first sentence of Hosoya Polynomial is $\left(9 k^{2}-3 k\right) x^{1}$. 
II. The first sentence of Schultz Polynomial is $\left(54 k^{2}-30 k\right) x^{1}$.

III. The first sentence of Modified Schultz Polynomial is $\left(81 k^{2}-63 k+6\right) x^{1}$.

$I V$. The second sentence of Hosoya Polynomial is $6 k(3 k-2) x^{2}$.

$V$. The second sentence of Schultz Polynomial is $\left(108 k^{2}-96 k+12\right) x^{2}$.

VI. The second sentence of Modified Schultz Polynomial is $\left(162 k^{2}-174 k+30\right) x^{2}$. VII. The third sentence of Hosoya Polynomial is $\left(27 k^{2}-27 k+3\right) x^{3}$.

VIII. The third sentence of Schultz Polynomial is $\left(162 k^{2}-192 k+30\right) x^{3}$.

$I X$. The third sentence of Modified Schultz Polynomial is $\left(243 k^{2}-333 k+75\right) x^{3}$.

It is obvious that, the farthest distance between two vertices of $H_{k}(k \geq 2)$ is equal to $4 k-1$. The notation of this terminology is $d(G)$ in graph theory. In other words, the diameter $d\left(H_{k}\right)$ of circumcoronene series of benzenoid is $4 k-1$, (by simple induction on $d\left(H_{k+1}\right)=d\left(H_{k}\right)+4$ and its first terms are $d\left(H_{1}=C_{6}\right)=3, d\left(H_{2}\right)=7, d\left(H_{3}\right)=11, \ldots$ see $\left.[17]\right)$.

Theorem 1.6. Let $H_{k}(\forall k \geq 2)$ be the circumcoronene series of benzenoid, with diameter $d\left(H_{k}\right)=4 k-1$. Then:

I. The latest sentence of Hosoya Polynomial is $\left(3 k^{2}\right) x^{4 k-1}$.

II. The latest sentence of Schultz Polynomial is $(2+2)\left(3 k^{2}\right) x^{4 k-1}$.

III. The latest sentence of Modified Schultz Polynomial is $(2 \times 2)\left(3 k^{2}\right) x^{4 k-1}$.

$I V$. The $4 k-2$ th sentence $\left(6 k^{2}+6 k-6\right) x^{4 k-2}$ of Hosoya Polynomial.

$V$. The $4 k-2$ th sentence $\left(30 k^{2}+18 k-24\right) x^{4 k-2}$ of Schultz Polynomial.

VI. The $4 k-2$ th sentence $\left(36 k^{2}+12 k-24\right) x^{4 k-2}$ of Modified Schultz Polynomial.

\section{Main results}

Before prove of Theorem 1.2, Theorem 1.3, Theorem 1.4, and Theorem 1.5 for molecular graph circumcoronene series of benzenoid $H_{k}$, we need the following denotation of $H_{3}$. Suppose $\mathbb{Z}_{6}=\{0,1, \ldots, 5\}$ is the cycle finite group of order 6 .

Denotation 2.1. Let $V(G)$ is the vertex set of $G=H_{3}$ with cardinality 54 and $E(G)$ is the edge set of $G$ with cardinality $\frac{36 \times 3+18 \times 2}{2}=72$.

Therefore, we name each vertex of $G$ by automorphism $f$ ( $f$ is bijection), such that:

$$
f: V(G) \longrightarrow\left\{v_{i}, u_{i}, x_{i}, y_{i}, z_{i}, t_{i}, a_{i}, b_{i}, c_{i} \mid i \in \mathbb{Z}_{6}\right\}
$$

and also,

$f: E(G) \longrightarrow\left\{v_{i} v_{i+1}, v_{i} u_{i}, u_{i} x_{i}, u_{i} y_{i}, x_{i} y_{i+1}, x_{i} z_{i}, y_{i} t_{i}, z_{i} a_{i}, z_{i} c_{i}, t_{i} b_{i}, t_{i} c_{i}, a_{i} b_{i+1} \mid i \in \mathbb{Z}_{6}\right\}$.

According to the Figure 2, we have the vertices $v_{i}, u_{i}, x_{i}, y_{i}, z_{i}, t_{i}$ as degree 3 and vertices $a_{i}, b_{i}, c_{i}$ as degree 2 , for all $i \in \mathbb{Z}_{6}$.

Definition 2.2. Let $d(u, v)=i$ is distance between vertices $u$ and $v$ of $G$. Then, $D_{i}\left(d_{1}, d_{2}\right)=\left\{(u, v) \mid u, v \in V(G), d(u, v)=i, d_{1}=\delta_{u} \& d_{2}=\delta_{v}\right\}$ is the set of dual $(u, v)$ of vertex set $V(G)$.

So, we have two sentences $\left|D_{i}\left(d_{1}, d_{2}\right)\right| \times x^{d_{1}+d_{2}}$ and $\left|D_{i}\left(d_{1}, d_{2}\right)\right| \times x^{d_{1} \cdot d_{2}}$ of the Schultz polynomial and the Modified Schultz polynomial respectively, such that 
$\forall u, v \in D_{i}\left(d_{1}, d_{2}\right), d_{1}+d_{2}=\delta_{u}+\delta_{u}$ and $d_{1} \cdot d_{2}=\delta_{u} \times \delta_{u}$.

Obviously, if $G=H_{k}$, we have

$$
D_{i}=\left\{(u, v) \mid u, v \in V\left(H_{3}\right), d(u, v)=i\right\}=D_{i}(3,3) \cup D_{i}(3,2) \cup D_{i}(2,2)
$$

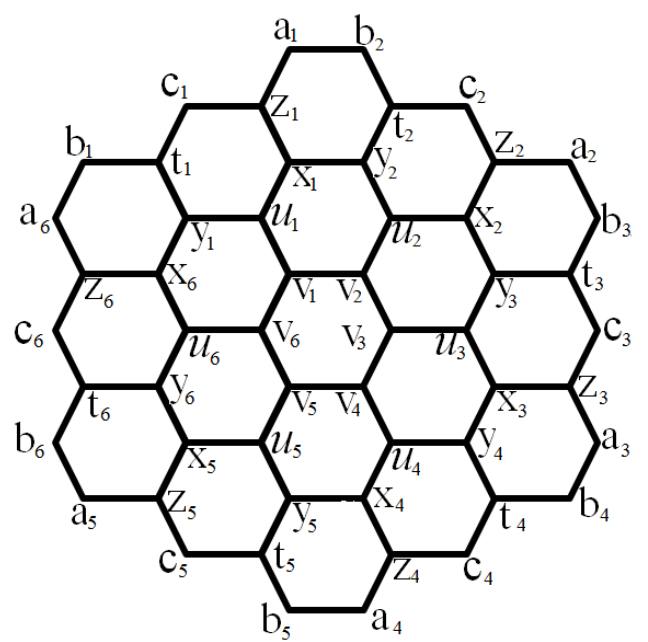

Figure 2. The circumcoronene $H_{3}$, that named by bijection $f$.

Proof. Let $G=H_{3}$ be the third member of Circumcoronene Series of Benzenoid. Since, there exist the number of 54 distinct vertices. Thus, we have the $\left(\begin{array}{l}54 \\ 2\end{array}\right)=$ 1431 distinct shortest path between vertices $u$ and $v$ of $G$.

Also, $\forall u, v \in V(G), \exists d(u, v) \in\{1,2, \ldots, 11\}$ and we will have eleven partitions for proof, by according to the Figure 2 .

I- If $d(u, v)=1$, then $D_{1}=E(G)$. So, we have three subsets of it.

I- 1. $D_{1}(3,3)=\left\{\left(v_{i}, v_{i+1}\right),\left(v_{i}, u_{i}\right),\left(u_{i}, x_{i}\right),\left(u_{i}, y_{i}\right),\left(x_{i}, y_{i+1}\right),\left(x_{i}, z_{i}\right),\left(y_{i}, t_{i}\right) \mid i \in\right.$ $\left.\mathbb{Z}_{6}\right\}$, therefore $\left|D_{1}(3,3)\right|=7 \times 6=42$. Also, $\delta_{v}+\delta_{u}=6$ and $\delta_{v} \times \delta_{u}=9$, because $\forall u, v \in D_{1}(3,3), \delta_{v}=\delta_{u}=3$. So, we have tow sentences $252 x^{1}, 378 x^{1}$ of the Schultz polynomial and Modified Schultz polynomial, respectively.

$I-2$. $D_{1}(3,2)=\left\{\left(z_{i}, a_{i}\right),\left(z_{i}, c_{i}\right),\left(t_{i}, b_{i}\right),\left(t_{i}, c_{i+1}\right) \mid i \in \mathbb{Z}_{6}\right\}$, therefore $\left|D_{1}(3,2)\right|=$ $4 \times 6=24$. Also, $\delta_{v}+\delta_{u}=5$ and $\delta_{v} \times \delta_{u}=6$. Since $\forall u, v \in D_{1}(3,2), \delta_{u}=2 \&$ $\delta_{v}=3$. So, we have tow sentences $120 x^{1}, 144 x^{1}$ of the Schultz polynomial and Modified Schultz polynomial, respectively.

$I-3$. Since $\left(a_{i}, b_{i+1}\right) \in D_{1}(2,2), \delta_{a_{i}}=\delta_{b_{i+1}}=2$. Hence, we have $24 x^{1}$.

So generally, first term of the Schultz polynomial is $396 x^{1}$ and first term of the Modified Schultz polynomial is $546 x^{1}$.

Now, we list all existent sets $D_{d}\left(d_{1}, d_{2}\right), \forall i \in \mathbb{Z}_{6}, d=2, \ldots, 11$ and $d_{1}, d_{2}=2$ or 3 , as follow:

$I I-D_{2}(3,3)=\left\{\left(v_{i}, v_{i+2}\right),\left(v_{i}, u_{i+1}\right),\left(v_{i}, u_{i-1}\right),\left(v_{i}, x_{i}\right),\left(v_{i}, y_{i}\right),\left(u_{i}, x_{i-1}\right),\left(u_{i}, y_{i+1}\right)\right.$, $\left.\left(u_{i}, z_{i}\right),\left(u_{i}, t_{i}\right),\left(x_{i}, y_{i}\right),\left(x_{i}, t_{i+1}\right),\left(y_{i}, z_{i-1}\right),\left(z_{i}, t_{i}\right)\right\}$ 
$D_{2}(3,2)=\left\{\left(x_{i}, a_{i}\right),\left(x_{i}, c_{i}\right),\left(y_{i}, b_{i}\right),\left(y_{i}, c_{i}\right),\left(z_{i}, b_{i+1}\right),\left(t_{i}, a_{i-1}\right)\right\}$

$D_{2}(2,2)=\left\{\left(a_{i}, c_{i}\right),\left(b_{i}, c_{i}\right)\right\}$

III- $D_{3}(3,3)=\left\{\left(v_{i}, v_{i+3}\right),\left(v_{i}, u_{i+2}\right),\left(v_{i}, u_{i-2}\right),\left(v_{i}, x_{i-1}\right),\left(v_{i}, y_{i-1}\right),\left(v_{i}, x_{i+1}\right)\right.$,

$\left(v_{i}, y_{i+1}\right),\left(v_{i}, z_{i}\right),\left(\bar{v}_{i}, t_{i}\right),\left(u_{i}, u_{i+1}\right),\left(u_{i}, t_{i+1}\right),\left(u_{i}, z_{i-1}\right),\left(y_{i}, z_{i}\right),\left(y_{i}, y_{i+1}\right)$,

$\left.\left(x_{i}, x_{i+1}\right),\left(x_{i}, t_{i}\right),\left(z_{i}, t_{i+1}\right)\right\}$

$D_{3}(3,2)=\left\{\left(u_{i}, a_{i}\right),\left(u_{i}, b_{i}\right),\left(u_{i}, c_{i}\right),\left(x_{i}, b_{i+1}\right),\left(x_{i}, c_{i+1}\right),\left(y_{i}, a_{i-1}\right),\left(y_{i}, c_{i-1}\right)\right.$,

$\left.\left(z_{i}, b_{i}\right),\left(t_{i}, a_{i}\right)\right\}$

$D_{3}(2,2)=\left\{\left(a_{i}, c_{i+1}\right),\left(b_{i}, c_{i-1}\right)\right\}$

$I V-D_{4}(3,3)=\left\{\left(v_{i}, u_{i+3}\right),\left(v_{i}, x_{i+2}\right),\left(v_{i}, x_{i-2}\right),\left(v_{i}, y_{i+2}\right),\left(v_{i}, y_{i-2}\right),\left(v_{i}, t_{i+1}\right)\right.$,

$\left(v_{i}, z_{i+1}\right),\left(v_{i}, t_{i-1}\right),\left(v_{i}, z_{i-1}\right),\left(u_{i}, u_{i+2}\right),\left(u_{i}, x_{i+1}\right),\left(u_{i}, y_{i-1}\right),\left(x_{i}, y_{i+2}\right)$,

$\left.\left(x_{i}, z_{i-1}\right),\left(x_{i}, z_{i+1}\right),\left(y_{i}, t_{i-1}\right),\left(y_{i}, t_{i+1}\right)\right\}$

$D_{4}(3,2)=\left\{\left(v_{i}, a_{i}\right),\left(v_{i}, b_{i}\right),\left(v_{i}, c_{i}\right),\left(u_{i}, b_{i+1}\right),\left(u_{i}, c_{i+1}\right),\left(u_{i}, a_{i-1}\right),\left(u_{i}, c_{i-1}\right)\right.$,

$\left.\left(x_{i}, b_{i}\right),\left(y_{i}, a_{i}\right),\left(t_{i}, b_{i+1}\right),\left(t_{i}, c_{i-1}\right),\left(z_{i}, a_{i-1}\right),\left(z_{i}, c_{i+1}\right)\right\}$

$D_{4}(2,2)=\left\{\left(a_{i}, b_{i}\right) d\left(a_{i}, b_{i}\right)\right\}$

$V-D_{5}(3,3)=\left\{\left(v_{i}, y_{i+3}\right),\left(v_{i}, x_{i+3}\right),\left(v_{i}, t_{i+2}\right),\left(v_{i}, t_{i-2}\right),\left(v_{i}, z_{i+2}\right),\left(v_{i}, z_{i-2}\right)\right.$,

$\left(u_{i}, u_{i+3}\right),\left(u_{i}, x_{i+2}\right),\left(u_{i}, x_{i-2}\right),\left(u_{i}, y_{i+2}\right),\left(u_{i}, y_{i-2}\right),\left(u_{i}, z_{i+1}\right),\left(u_{i}, t_{i-1}\right)$,

$\left.\overline{\left(x_{i}, y_{i-1}\right)},\left(x_{i}, t_{i+2}\right),\left(t_{i}, t_{i+1}\right),\left(z_{i}, z_{i+1}\right),\left(y_{i}, z_{i-2}\right)\right\}$

$D_{5}(3,2)=\left\{\left(v_{i}, a_{i+1}\right),\left(v_{i}, b_{i+1}\right),\left(v_{i}, c_{i+1}\right),\left(v_{i}, a_{i-1}\right),\left(v_{i}, b_{i-1}\right),\left(v_{i}, c_{i-1}\right)\right.$,

$\left.\left(x_{i}, a_{i+1}\right),\left(x_{i}, a_{i-1}\right),\left(x_{i}, c_{i-1}\right),\left(y_{i}, b_{i+1}\right),\left(y_{i}, b_{i-1}\right),\left(y_{i}, c_{i+1}\right)\right\}$

$D_{5}(2,2)=\left\{\left(a_{i}, a_{i+1}\right),\left(b_{i}, b_{i+1}\right),\left(c_{i}, c_{i+1}\right)\right\}$

$V I-D_{6}(3,3)=\left\{\left(v_{i}, t_{i+3}\right),\left(v_{i}, z_{i+3}\right),\left(u_{i}, y_{i+3}\right),\left(u_{i}, x_{i+3}\right),\left(u_{i}, t_{i+2}\right),\left(u_{i}, t_{i-2}\right)\right.$,

$\left.\left(u_{i}, z_{i+2}\right),\left(u_{i}, z_{i-2}\right),\left(x_{i}, x_{i+2}\right),\left(x_{i}, y_{i-2}\right),\left(x_{i}, t_{i-1}\right),\left(y_{i}, y_{i+2}\right),\left(y_{i}, z_{i+1}\right),\left(z_{i}, t_{i+2}\right)\right\}$

$D_{6}(3,2)=\left\{\left(v_{i}, a_{i+2}\right),\left(v_{i}, b_{i+2}\right),\left(v_{i}, c_{i+2}\right),\left(v_{i}, a_{i-2}\right),\left(v_{i}, b_{i-2}\right),\left(v_{i}, c_{i-2}\right)\right.$,

$\left(u_{i}, a_{i-1}\right),\left(u_{i}, b_{i+1}\right),\left(x_{i}, b_{i+2}\right),\left(x_{i}, c_{i+2}\right),\left(y_{i}, a_{i-2}\right),\left(y_{i}, c_{i-2}\right),\left(z_{i}, c_{i-1}\right)$,

$\left.\left(z_{i}, a_{i+1}\right),\left(t_{i}, b_{i-1}\right),\left(t_{i}, c_{i+1}\right)\right\}$

$D_{6}(2,2)=\left\{\left(a_{i}, b_{i+2}\right)\right\}$

$\operatorname{VII-D} D_{7}(3,3)=\left\{\left(u_{i}, t_{i+3}\right),\left(u_{i}, z_{i+3}\right),\left(x_{i}, x_{i+3}\right),\left(x_{i}, y_{i+3}\right),\left(x_{i}, z_{i+2}\right),\left(x_{i}, z_{i-2}\right)\right.$,

$\left.\left(x_{i}, t_{i-2}\right),\left(y_{i}, y_{i+3}\right),\left(y_{i}, t_{i+2}\right),\left(y_{i}, t_{i-2}\right), \overline{\left(y_{i}, z_{i+2}\right)},\left(z_{i}, t_{i-1}\right)\right\}$

$D_{7}(3,2)=\left\{\left(v_{i}, a_{i+3}\right),\left(v_{i}, b_{i+3}\right),\left(v_{i}, c_{i+3}\right),\left(u_{i}, a_{i+2}\right),\left(u_{i}, b_{i+2}\right),\left(u_{i}, c_{i+2}\right)\right.$,

$\left(u_{i}, a_{i-2}\right),\left(u_{i}, b_{i-2}\right),\left(u_{i}, c_{i-2}\right),\left(x_{i}, b_{i-1}\right),\left(y_{i}, a_{i+1}\right),\left(z_{i}, b_{i+2}\right),\left(z_{i}, c_{i+2}\right)$,

$\left.\left(t_{i}, a_{i-2}\right),\left(t_{i}, c_{i-2}\right)\right\}$

$D_{7}(2,2)=\left\{\left(a_{i}, c_{i-1}\right),\left(b_{i}, c_{i+1}\right)\right\}$

VIII- $D_{8}(3,3)=\left\{\left(x_{i}, t_{i+3}\right),\left(x_{i}, z_{i+3}\right),\left(y_{i}, t_{i+3}\right),\left(y_{i}, z_{i+3}\right),\left(z_{i}, z_{i+2}\right),\left(z_{i}, t_{i-2}\right)\right.$,

$\left.\left(t_{i}, t_{i+2}\right)\right\}$

$D_{8}(3,2)=\left\{\left(u_{i}, a_{i+3}\right),\left(u_{i}, b_{i+3}\right),\left(u_{i}, c_{i+3}\right),\left(x_{i}, a_{i+2}\right),\left(x_{i}, a_{i-1}\right),\left(x_{i}, b_{i-1}\right)\right.$,

$\left(x_{i}, c_{i-1}\right),\left(y_{i}, a_{i+2}\right),\left(y_{i}, b_{i+2}\right),\left(y_{i}, b_{i-1}\right),\left(y_{i}, c_{i+2}\right),\left(z_{i}, b_{i-1}\right),\left(t_{i}, a_{i+1}\right\}$

$D_{8}(2,2)=\left\{\left(a_{i}, c_{i+2}\right),\left(b_{i}, c_{i-2}\right),\left(c_{i}, c_{i+2}\right)\right\}$

$I X-D_{9}(3,3)=\left\{\left(z_{i}, z_{i+3}\right),\left(z_{i}, t_{i+3}\right),\left(t_{i}, t_{i+3}\right)\right\}$

$D_{9}(3,2)=\left\{\left(x_{i}, a_{i+3}\right),\left(x_{i}, b_{i+3}\right),\left(x_{i}, c_{i+3}\right),\left(y_{i}, a_{i+3}\right),\left(y_{i}, b_{i+3}\right),\left(y_{i}, c_{i+3}\right)\right.$,

$\left.\left(z_{i}, a_{i+2}\right),\left(z_{i}, a_{i-2}\right),\left(z_{i}, b_{i-2}\right),\left(z_{i}, c_{i-2}\right),\left(t_{i}, a_{i+2}\right),\left(t_{i}, b_{i+2}\right),\left(t_{i}, b_{i-2}\right),\left(t_{i}, c_{i+2}\right)\right\}$

$D_{9}(2,2)=\left\{\left(a_{i}, b_{i-1}\right)\right\}$

$X-D_{10}(3,2)=\left\{\left(z_{i}, a_{i+3}\right),\left(z_{i}, b_{i+3}\right),\left(z_{i}, c_{i+3}\right),\left(t_{i}, a_{i+3}\right),\left(t_{i}, b_{i+3}\right),\left(t_{i}, c_{i+3}\right)\right\}$

$D_{10}(2,2)=\left\{\left(a_{i}, a_{i+2}\right),\left(a_{i}, b_{i-2}\right),\left(a_{i}, c_{i-2}\right),\left(b_{i}, b_{i+2}\right),\left(b_{i}, c_{i+2}\right)\right\}$ 


$$
X I-D_{11}=\left\{\underline{\left(a_{i}, a_{i+3}\right)},\left(a_{i}, b_{i+3}\right),\left(a_{i}, c_{i+3}\right), \underline{\left(b_{i}, b_{i+3}\right)},\left(b_{i}, c_{i+3}\right), \underline{\left(c_{i}, c_{i+3}\right)}\right\}
$$

Therefore, by use of Part I, Part II and above sets, we digest all computation in following table.

Now, we equation all distance of any $u, v \in V(G)$. Also, we obtain the favorite

TABLE 1 . The existent sets $D_{d}\left(d_{1}, d_{2}\right)$, for every $d=2, \ldots, 11$ and $d_{1}, d_{2}=2$ or 3 , where $i=d_{v}+d_{u} \& j=d_{v} \times d_{u}$

\begin{tabular}{|c|c|c|c|}
\hline$\overline{d e(u, v)}$ & $i \& j$ & $\left|D_{d}\left(\delta_{v}, \delta_{u}\right)\right|$ & Coefficients of $x^{i}$ and $x^{j}$ \\
\hline \multirow[t]{3}{*}{ 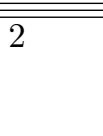 } & "6\&9 & $\overline{136=78}$ & $4 \quad 468 \& 702$ \\
\hline & $5 \& 6$ & $66=36$ & $180 \& 216$ \\
\hline & $4 \& 4$ & $26=12$ & $48 \& 48$ \\
\hline \multirow[t]{3}{*}{3} & $6 \& 9$ & $3+166=99$ & $594 \& 891$ \\
\hline & $5 \& 6$ & $96=54$ & $270 \& 324$ \\
\hline & $4 \& 4$ & $26=12$ & $48 \& 48$ \\
\hline \multirow[t]{3}{*}{4} & $6 \& 9$ & $176=102$ & $612 \& 918$ \\
\hline & $5 \& 6$ & $136=78$ & $390 \& 468$ \\
\hline & $4 \& 4$ & 6 & $24 \& 24$ \\
\hline \multirow[t]{3}{*}{5} & $6 \& 9$ & $3+176=105$ & $630 \& 945$ \\
\hline & $5 \& 6$ & $126=72$ & $360 \& 432$ \\
\hline & $4 \& 4$ & $36=18$ & $72 \& 72$ \\
\hline \multirow[t]{3}{*}{6} & $6 \& 9$ & $146=84$ & $504 \& 756$ \\
\hline & $5 \& 6$ & $166=96$ & $480 \& 576$ \\
\hline & $4 \& 4$ & 6 & $24 \& 24$ \\
\hline \multirow[t]{3}{*}{7} & $6 \& 9$ & $6+106=66$ & $396 \& 594$ \\
\hline & $5 \& 6$ & $156=90$ & $450 \& 540$ \\
\hline & $4 \& 4$ & $26=12$ & $48 \& 48$ \\
\hline \multirow[t]{3}{*}{8} & $6 \& 9$ & $76=42$ & $252 \& 378$ \\
\hline & $5 \& 6$ & $136=78$ & $390 \& 468$ \\
\hline & $4 \& 4$ & $36=18$ & $72 \& 72$ \\
\hline \multirow[t]{3}{*}{9} & $6 \& 9$ & $6+6=12$ & $72 \& 108$ \\
\hline & $5 \& 6$ & $146=84$ & $420 \& 504$ \\
\hline & $4 \& 4$ & 6 & $24 \& 24$ \\
\hline \multirow[t]{2}{*}{10} & $5 \& 6$ & $66=36$ & $180 \& 216$ \\
\hline & $4 \& 4$ & $56=30$ & $120 \& 120$ \\
\hline 11 & $4 \& 4$ & $36+9=27$ & $108 \& 108$ \\
\hline
\end{tabular}

polynomial and their indices. Thus, the Schultz polynomial of $H_{3}$ is equal to:

$$
\begin{aligned}
& S c\left(H_{3}, x\right)=396 x+696 x^{2}+912 x^{3}+1026 x^{4}+1062 x^{5} \\
& +1008 x^{6}+894 x^{7}+714 x^{8}+516 x^{9}+300 x^{10}+108 x^{11}
\end{aligned}
$$

And Schultz index of $H_{3}$ is $S c\left(H_{3}\right)=40788$. 
The Modified Schultz polynomial of $H_{3}$ is equal to:

$$
\begin{aligned}
& S c^{*}\left(H_{3}, x\right)=546 x+966 x^{2}+1263 x^{3}+1410 x^{4}+1449 x^{5} \\
& +1356 x^{6}+1182 x^{7}+918 x^{8}+336 x^{9}+336 x^{10}+108 x^{11}
\end{aligned}
$$

Also the Modified Schultz index of $H_{3}$ is $S c^{*}\left(H_{3}\right)=53502$. Here, we complete the proof of Theorem 1.2.

Proof. Let $G=H_{3}$ be the third member of circumcoronene series of benzenoid. According to Definition 2.2, since $D_{i}=D_{i}(3,3) \cup D_{i}(3,2) \cup D_{i}(2,2)$ $(\forall i \in\{1,2, \ldots, 11\})$ and $d(G, i)=\left|D_{i}\right|=\left|D_{i}(3,3)\right|+\left|D_{i}(3,2)\right|+\left|D_{i}(2,2)\right|$. Also, $H(G, x)=\sum_{i=0}^{d(G)} d(G, i) x^{i}$.

Now by reference to the proof of Theorem 1.3, the Hosoya polynomial of $\mathrm{H}_{3}$ is:

$$
\begin{gathered}
H\left(H_{3}, x\right)=\sum_{i=0}^{d\left(H_{3}\right)} d\left(H_{3}, i\right) x^{i}=54+72 x+126 x^{2}+165 x^{3} \\
+186 x^{4}+195 x^{5}+186 x^{6}+168 x^{7}+138 x^{8}+102 x^{9}+66 x^{10}+27 x^{11} .
\end{gathered}
$$

Hence The Wiener index of benzenoid $H_{3}$ is equal to:

$$
W\left(H_{3}\right)=\sum_{i=0}^{d\left(H_{3}\right)} i . d\left(H_{3}, i\right)=7809 .
$$

It is obvious that, $H\left(H_{3}, 1\right)=1431+54=\left(\begin{array}{c}n \\ 2\end{array}\right)+n$.

Here, the proof of Theorem 1.3 was completed.

Before proof of Theorem 1.4, we introduce the general notation for vertices of circumcoronene series of benzenoid $H_{k}$, in following denotation.

Denotation 2.3. Let $V(G)$ is the vertex set of $G=H_{k}$ with cardinality $6 k^{2}$. So, we name all vertices of degree 2 by $\gamma_{i}$ (for $i=1, \ldots, 6 k$ ) and say family $\boldsymbol{\Gamma}$, such that their adjacent vertices named by $\beta_{i}$ (for $i=1, \ldots, 6 k-$ 1 and $i \neq k, 2 k, 3 k, 4 k, 5 k)$ and say family $\mathbf{B}$. In other words, $D_{1}(3,2)=$ $\left\{\left(\gamma_{i}, \beta_{i}\right),\left(\gamma_{i-1}, \beta_{i}\right) \mid i=1, \ldots, 6 k-6\right\}$ and $D_{1}(2,2)=\left\{\left(\gamma_{j k+1}, \gamma_{(j+1) k}\right) \mid j \in \mathbb{Z}_{6}\right\}$. Also $\forall i$, we name all adjacent vertices with $\beta_{i}$, by $\alpha_{i}$ (say family $\mathbf{A}$ ). $\alpha_{i}$ adjacent vertices named by $x_{i}$ (for $i=1, \ldots, 6 k-1$ and $i \neq k, 2 k, 3 k, 4 k, 5 k$ ) and say family $\mathbf{X}$. We name another vertices by $y_{i} i=1, \ldots, 6 k^{2}-24 k+24$, respectively and say family $\mathbf{Y}$. See Figure 3.

Proof. ( $i)$. Let $E\left(H_{k}\right)(\forall k \geq 2)$ is the edge set of circumcoronene series of benzenoid, since $\left|E\left(H_{k}\right)\right|=\overline{9} k^{2}-3 k$, it is obvious that the first sentence of $H\left(H_{k}\right)$ is $\left(9 k^{2}-3 k\right) x^{1}$. Also, by according to Denotation $2.2\left|D_{1}(2,2)\right|=$ $\left|\left\{\left(\gamma_{j k+1}, \gamma_{(j+1) k}\right) \mid j \in \mathbb{Z}_{6}\right\}\right|=6,\left|D_{1}(3,2)\right|=\mid\left\{\left(\gamma_{i}, \beta_{i}\right),\left(\gamma_{i-1}, \beta_{i}\right) \mid i=1, \ldots, 6 k-\right.$ $6\} \mid=12(k-1)$, then $\left|D_{1}(3,3)\right|=\left|E\left(H_{k}\right)\right|-\left|D_{1}(2,2)\right|-\left|D_{1}(3,2)\right|=9 k^{2}-15 k+6$. So the coefficient of first sentence of Schultz Polynomial is $4 \times 6+5 \times 12(k-$ $1)+6 \times\left(9 k^{2}-15 k+6\right)=54 k^{2}-30 k$. By similar argument the coefficient of first sentence of Modified Schultz Polynomial of $H_{k}$ will be $243 k^{2}-333 k+75$. 
604

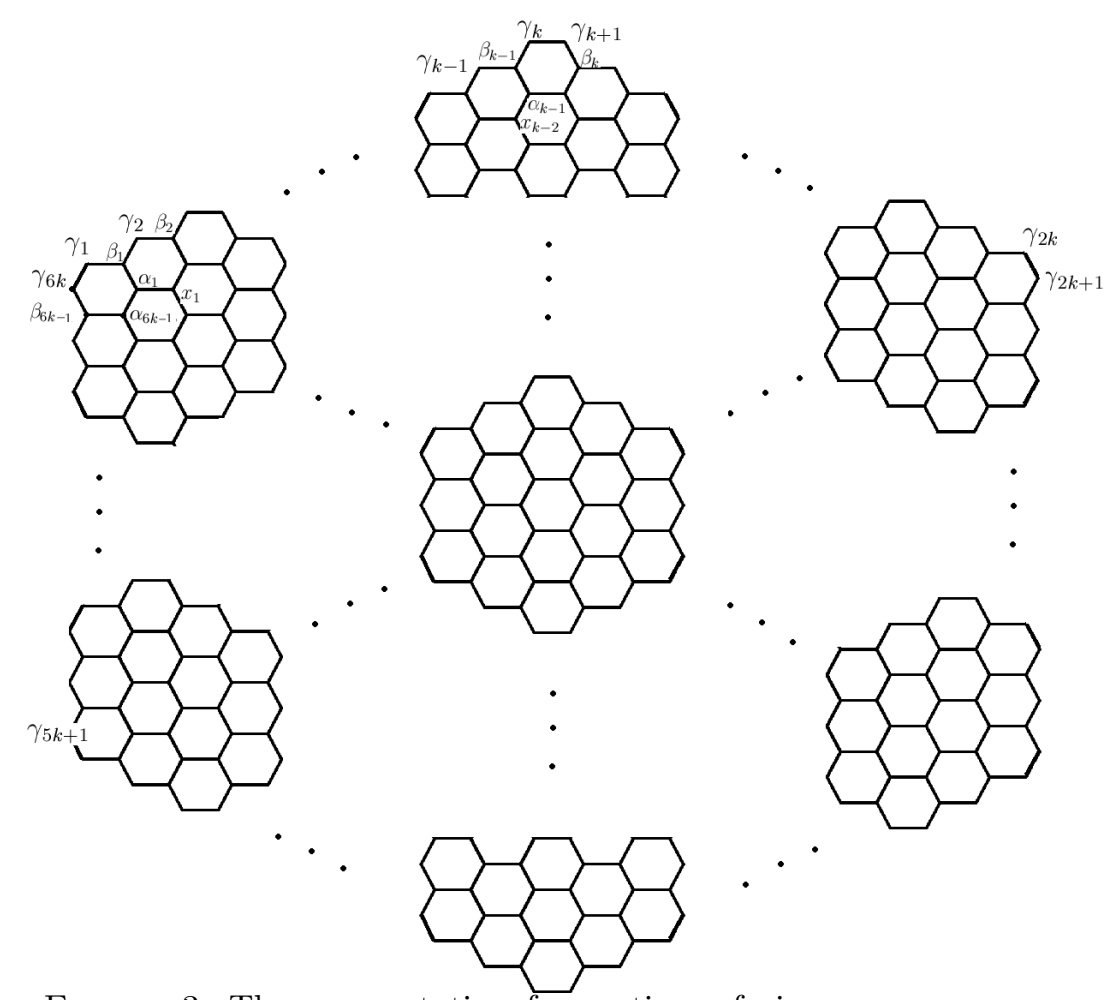

FIGURE 3. The new notation for vertices of circumcoronene series of benzenoid $H_{k}$.

(ii). We see that there exist six paths with length 2 for all $C_{6}$ (the cycle of order 6$)$ and also $6(k-1)$ paths with length 2 between vertices $\gamma_{j k+i}, \gamma_{j k+i+1}$ (for $i=1, \ldots, k-1$ and $j=0,1,2,3,4,5$ ), by according to the Figure 4 . Now, we denote the number of $C_{6}$ in the molecular graph $G=H_{k}$ by $\zeta_{k}$. It is obvious that, $\zeta_{k}=\zeta_{k-1}+6(k-1)$. Thus $\zeta_{k}=\zeta_{1}+6 \sum_{2}^{k}(i-1)=\zeta_{1}+6 \sum_{1}^{k-1} i=3 k(k-1)+1$. So, the second sentence of Hosoya Polynomial is $[6(3 k(k-1)+1)+6(k-1)] x^{2}=$ $[6 k(3 k-2)] x^{2}$.

Also, $\left|D_{2}(3,2)\right|=\left|\left\{\left(\gamma_{i}, \alpha_{i+1}\right),\left(\gamma_{i}, \alpha_{i-1}\right) \mid i \in \mathbb{Z}_{6 k}\right\}\right|=6 \times 2 k=12 k,\left|D_{2}(2,2)\right|=$ $6(k-1)$ and $\left|D_{2}(3,3)\right|=6(3 k(k-1)+1)-12 k=18 k^{2}-30 k+6$ by according to the Figure 4.

Therefore, the second sentence of Schultz Polynomial and Modified Schultz Polynomial of $H_{k}$ will be $\left[4 \times 6(k-1)+5 \times 12 k+6 \times\left(18 k^{2}-30 k+6\right)\right] x^{2}=\left(108 k^{2}-96 k+\right.$ $12) x^{2}$ and $\left[4 \times 6(k-1)+6 \times 12 k+9 \times\left(18 k^{2}-30 k+6\right)\right] x^{2}=\left(162 k^{2}-174 k+30\right) x^{2}$, respectively.

(iii). By according to the Figure 5, Figure 6 and use of Denotation 2.2 for circumcoronene series of benzenoid $H_{k}(\forall k \geq 2)$, we categorize the path with length 3 , in based their first point, their end point and listed in the following table.

Also by according to the Definition 2.2, we have $D_{i}\left(d_{1}, d_{2}\right)=\{(u, v) \mid u, v \in$ $\left.V(G), d(u, v)=i, d_{1}=\delta_{u} \& d_{2}=\delta_{v}\right\}$. So, we write $D_{3}(3,3)=\{(u, v) \mid u, v \in$ 


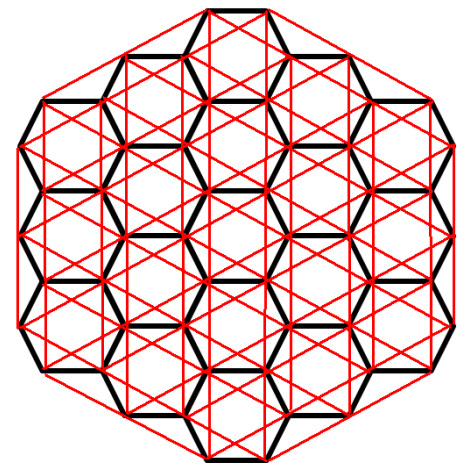

Figure 4. Example: The path with length 2 of circumcoronene $H_{3}$.

TABLE 2. Categorization the path with length 3 , in based their first and end point, and the number of them.

\begin{tabular}{l|cc}
\hline \hline The first point & The families of end points & The number of these path \\
\hline \hline $\begin{array}{l}\gamma_{i k+1} \text { and } \gamma_{(i+1) k} \\
(i=0,1, \ldots, 5)\end{array}$ & $\Gamma, B, A, X$ & $4 \times 12=48$ \\
$\gamma_{i k+2}$ and $\gamma_{(i+1) k-1}$ & $\Gamma, B, A, A, X$ & $5 \times 12=60$ \\
$(i=0,1, \ldots, 5)$ & & \\
$\gamma_{i k+j}(i=0,1, \ldots, 5$ & $\& j=3, \ldots, k-2)$ \\
$\begin{array}{l}\beta_{i k+1} \text { and } \beta_{(i+1) k-1} \\
(i=0,1, \ldots, 5)\end{array}$ & $B, B, A, A, X$ & $5 \times 6(k-2)=30 k-120$ \\
$\beta_{i k+j}(i=0,1, \ldots, 5$ & $\Gamma, B, A, X, Y$ & $5 \times 12=60$ \\
$\& j=2, \ldots, k-2)$ & $\Gamma, \Gamma, A, A, Y, Y$ & $6 \times 6(k-3)=36 k-108$ \\
$\alpha_{i k+1}$ and $\alpha_{(i+1) k-1}$ & & \\
$(i=0,1, \ldots, 5)$ & $\Gamma, \Gamma, B, A, X, Y, Y$ & $7 \times 12=84$ \\
$\alpha_{i k+j}(i=0,1, \ldots, 5$ & & \\
$\& j=2, \ldots, k-2)$ & $B, B, A, A, Y, Y, Y$ & $7 \times 6(k-3)=42 k-126$ \\
$x_{i} \in X$ & $\Gamma, \Gamma, B, A, A, X, Y, Y$ & $9 \times 6(k-2)=54 k-108$ \\
$y_{i} \in Y$ & All in $X$ or $Y$ & $9 \times 6(k-2)^{2}$ \\
& & $=54 k^{2}-216 k+216$ \\
\hline
\end{tabular}

$Y, X, A, B \& d(u, v)=3\}, D_{3}(3,2)=\{(u, v) \mid u \in Y, X, A, B \& v \in \Gamma, d(u, v)=3\}$ and $D_{3}(2,2)=\{(u, v) \mid u, v \in \Gamma, d(u, v)=3\}$ for $G=H_{k}$, by use of the Denotation 2.1. Thus, $\left|D_{3}(3,3)\right|,\left|D_{3}(3,2)\right|$ and $\left|D_{3}(2,2)\right|$ are equal to $\frac{54 k^{2}-114 k+54}{2}$, $\frac{60 k-72}{2}$ and $\frac{24}{2}$, respectively (see Table 1 ).

Now, we can calculate the third sentence of Hosoya Polynomial, Schultz polynomial and Modified Schultz polynomial of $G=H_{k}$. Therefore, we have three 
sentences

$$
\begin{gathered}
\left.\left[27 k^{2}-57 k+27+30 k-36+12\right)\right] x^{3}=\left(27 k^{2}-27 k+3\right) x^{3}, \\
{\left[6\left(27 k^{2}-57 k+27\right)+5(30 k-36)+4(12)\right] x^{3}=\left(162 k^{2}-192 k+30\right) x^{3}}
\end{gathered}
$$

and

$$
\left[9\left(27 k^{2}-57 k+27\right)+6(30 k-36)+4(12)\right] x^{3}=\left(243 k^{2}-333 k+75\right) x^{3}
$$

of Hosoya Polynomial, Schultz polynomial and Modified Schultz polynomial, respectivelv. Now we comnlete the proof of Theorem 1.4.
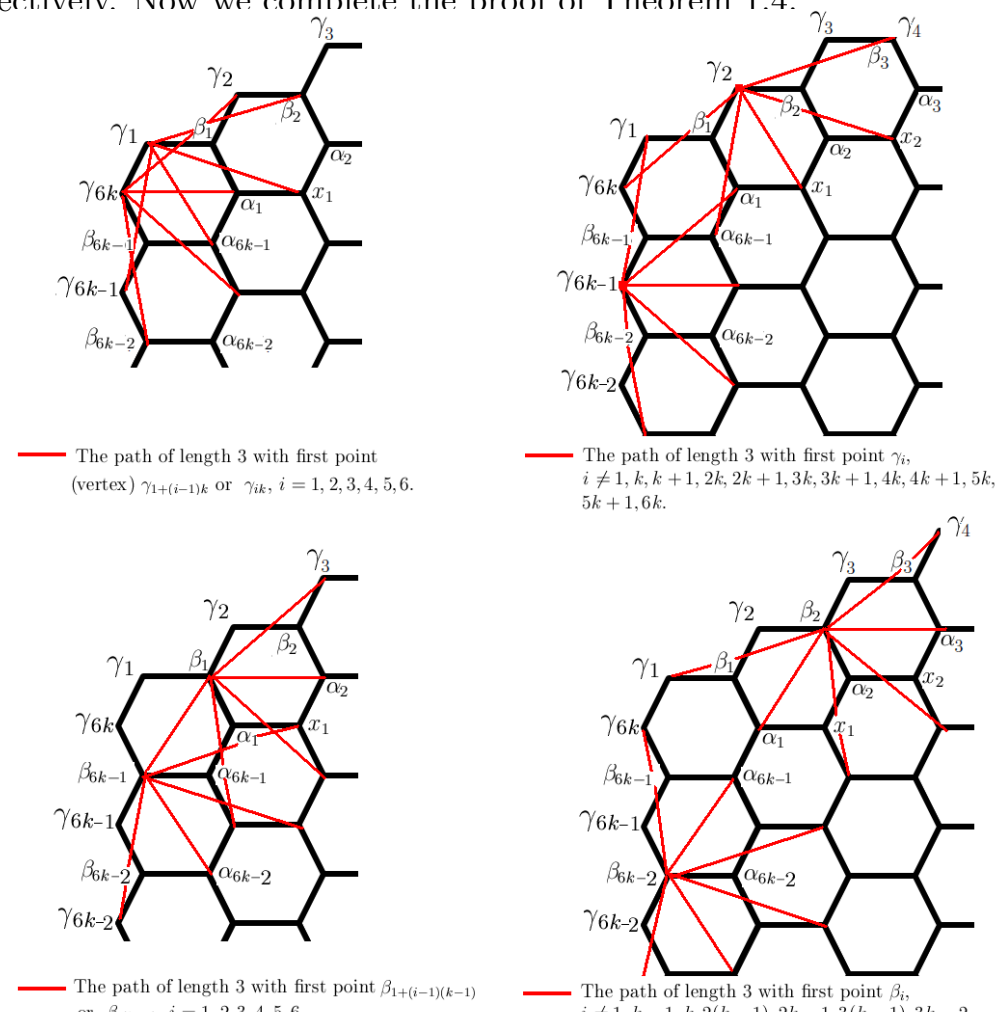
$5 k+1,6 k$

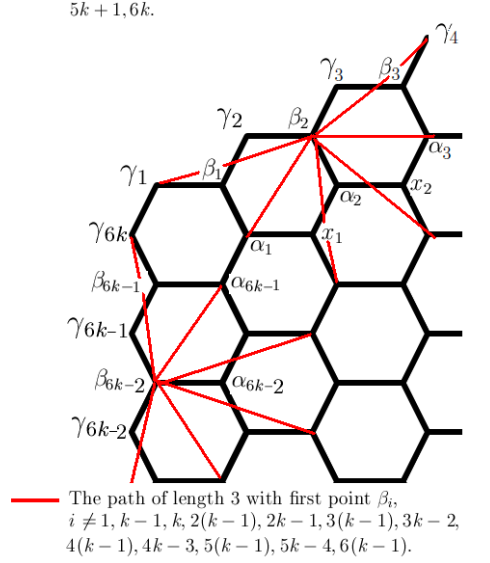

Figure 5. Example: The path with length 3 of circumcoronene series of benzenoid $H_{k}$, that their first point is $\gamma_{1}, \gamma_{2}, \beta_{1}$ and $\beta_{2}$.

Proof. $(i)$. By according to the Figure 3 and use the Denotation 2.2 for circumcoronene series of benzenoid $H_{k}(\forall k \geq 2)$, the distance $d\left(H_{k}\right)$ exist between some of the vertices with degree 2 . It is easy to see that, $\forall i \in \mathbb{Z}_{\Varangle}=\{0,1, \ldots, 5\}$ $\& \forall l, j \in\{1, \ldots, k\}, d\left(\gamma_{i k+j}, \gamma_{(i+3) k+l}\right)=4 k-1$ such that the index of $\gamma_{i} \in \Gamma$ exhibit from $\mathbb{Z}_{6 k}=\{1, \ldots, 6 k\}$. So, the latest sentence of Hosoya Polynomial of 

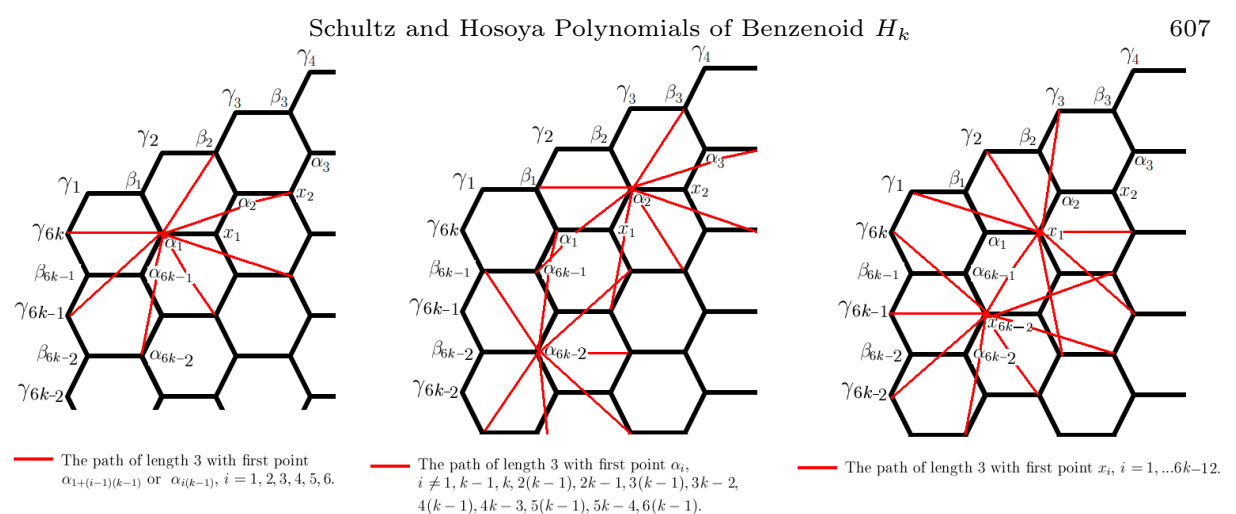

Figure 6. Example: The path with length 3 of circumcoronene series of benzenoid $H_{k}$, that their first point is $\alpha_{1}, \alpha_{2} x_{1}$.

circumcoronene series of benzenoid $H_{k}$ will be $\left(3 k^{2}\right) x^{4 k-1}$ and the latest sentence of Schultz polynomial and Modified Schultz polynomial of $H_{k}$ will be $12 k^{2} x^{4 k-1}$.

(ii). Also by according to the part $X$ of proof of Theorem 1.4, we see that there exist distance $4 k-2$ between vertices $\gamma_{i k+j}, \beta_{(i+3) k+l}$ and $d\left(\gamma_{i k+j}, \gamma_{(i+2) k+l}\right)=$ $d\left(\gamma_{i k+j}, \gamma_{(i+4) k+l}\right)=4 k-2, \forall i \in \mathbb{Z}_{\Varangle}=\{0,1, \ldots, 5\} \& \forall l, j \in\{1, \ldots, k\}$. Hence, we have $\left|D_{4 k-2}(2,2)\right|=6(k+k-1)$ and $\left|D_{4 k-1}(3,2)\right|=6 k(k-1)$. So, the coefficient of sentence $4 k-2$ th of Hosoya Polynomial is $6 k^{2}+6 k-6$ and we have coefficients $5\left(6 k^{2}-6 k\right)+4(12 k-6)=30 k^{2}+18 k-24$ and $6\left(6 k^{2}-6 k\right)+4(12 k-6)=$ $36 k^{2}+12 k-24$ of the Schultz polynomial and Modified Schultz polynomial, respectively.

\section{Conclusions}

In this paper, counting polynomials called "Schultz, Modified Schultz and Hosoya" of circumcoronene $H_{3}$ were determined and several sentence of favorite polynomials of circumcoronene series of benzenoid $H_{k}, k \geq 2$. See above theorems.

\section{Acknowledgments}

The author is thankful to Dr. Mehdi Alaeiyan and Seied Hamid Hosseini of Department of Mathematics, Iran University of Science and Technology (IUST), Prof. Mircea V. Diudea and Dr. Katalin Kata from Faculty of Chemistry and Chemical Engineering Babes-Bolyai University (Romania) and Prof. Ali Reza Ashrafi from Department of Mathematics of Faculty of Science of University of Kashan (Iran) for his precious support and suggestions.

\section{REFERENCES}

1. Y. Alizadeh, A. Iranmanesh and S. Mirzaie Computing Schultz Polynomial, Schultz Index of $C_{60}$ Fullerene By Gap Program, Digest. J. Nanomater. Bios 4(1) (2009), 7-10. 
2. A.R. Ashrafi and M. Ghorbani A Gap Program For Computing The Hosoya Polynomial and Wiener Index of Nano Structures, Digest. J. Nanomater. Bios 4(2) (2009), 389-393.

3. J. Devillers and A. Balaban, Gordon and Breech Amsterdam. (1999).

4. J.R. Dias From benzenoid hydrocarbons to fullerene carbons, MATCH Commun. Math. Comput. Chem 4 (1996), 57-85.

5. M.V. Diudea, Studia Univ. Babes-Bolyai 4 (2003), 3-21.

6. M.V. Diudea Hosoya polynomial in Tori, MATCH Commun. Math. Comput. Chem $\mathbf{4 5}$ (2002), 109-122.

7. A.A. Dobrynin, Croat. Chem. Acta 4 (1999), 869.

8. A. Dress and G. Brinkmann, MATCH Commun. Math. Comput. Chem 33 (1996), 87-100.

9. M. Eliasi and B. Taeri Schultz Polynomials of Composite Graphs, Appl. Anal. Discrete Math 2 (2008), 285-296.

10. M.R. Farahani Schultz polynomial, Modified Schultz polynomial, Hosoya polynomial and Wiener Index of Capra of planar benzenoid: $\mathrm{Ca}\left(C_{6}\right)$, Submited (2011).

11. M. Goldberg, J. Tohoku Math 43 (1937), 104-108.

12. I. Gutman, J. Chem. Inform. Comput. Sci 34 (1994), 1087.

13. I. Gutman and S. Klavźar, M. Petkovsek and P. Zigert On Hosoya Polynomial of benzenoid Graph

14. I. Gutman and S. Klavźar A method for calculationg Wiener numbers of benzenoid hydrocarbons and phenylenes, ACH Models Chem 133 (1996), 389-399.

15. O. Halakoo, O. Khormali and A. Mahmiani Bounds For Schultz Index of Pentachains, Digest. J. Nanomater. Bios 4(4) (2009), 687-691.

16. A. Heydari On The Modified Schultz Index of $C_{4} C_{8}(S)$ Nanotubes And Nanororus, Digest. J. Nanomater. Bios 5(1) (2010), 51-56.

17. H. Hosoya, Bull. Chem. Soc. Jpn 44 (1971), 23-32.

18. H. Hosoya On some counting polynomials in chemistry, Discrete Appl. Math 19 (1988), 239-257.

19. A. Iranmanesh and Y. Alizadeh Computing Hyper Wiener and Schultz Indices of $T U Z C_{6}[p, q]$ Nanotube By Gap Program, Digest. J. Nanomater. Bios 4(1) (2009), 607-611.

20. A. Iranmanesh and Y. Alizadeh Computing Szeged and Schultz Indices of $\mathrm{HAC}_{5} \mathrm{C}_{6} \mathrm{C}_{7}[p, q]$ Nanotube By Gap Program, Digest. J. Nanomater. Bios 4(1) (2009), 67-72.

21. S. Klavźar and I. Gutman, Disc. Appl. Math 80 (1997), 73.

22. S. Klavźar and I. Gutman Bounds for The Schultz Molecular Topological Index of benzenoid Systems in Terms of Wiener Index, J. Chem. Inf. Comput. Sci 37(4) (1997), 741-744.

23. S. Klavżar A Bird's Eye View of The Cut Method And A Survey of Its Applications In Chemical Graph Theory, MATCH Commun. Math. Comput. Chem 60 (2008), 255-274.

24. S. Klavźar and I. Gutman A Comparison of The Schultz Molecular Topological Index with The Wiener Index. J. Chem. Inf. Comput. Sci 36(5) (1996), 1001-1003.

25. H.P. Schultz, J. Chem. Inf. Comput. Sci 29 (1989), 227.

26. H.P. Schultz, J. Chem. Inf. Comput. Sci 40 (2000), 1158.

27. H. Shabani and A.R. Ashrafi Applications of The Matrix Package Matlab in Computing The Hosoya Polynomial of Zig-Zag Nanotubes, Digest. J. Nanomater. Bios 4(3) (2009), 423-428.

28. K. Xu Computing The Hosoya Index And The Wiener Index of An Infinite Class of Dendrimers, Digest. J. Nanomater. Bios 6(1) (2011), 265-270.

29. Sh. Xu and H. Zhang Generalized Hosoya Polynomials of Hexagonal Chains, J. Math. Chem. 43 (2008), 2.

\section{Mohammad Reza Farahani}

Department of Mathematics, Iran University of Science and Technology (IUST), Narmak, Tehran 16844, Iran.

e-mail: Mr_Farahani@MathDep.iust.ac.ir 